\title{
„Für die beste Entwicklung der Allerkleinsten“ - Frauenmilchbank-Initiative e.V.
}

FRAUENMILCHBANKINITIATIVE | F M B I

Muttermilch ist vor allem für Frühgeborene und kranke Neugeborene wichtig. Doch was tun, wenn die Milch der eigenen Mutter nicht ausreicht? Hier setzt die bundesweit tätige Frauenmilchbank-Initiative an. Frauenmilchbanken sammeln, untersuchen, lagern und verteilen gespendete Muttermilch an Babys, die von ihrer Mutter keine Milch erhalten können.

Stillen gilt als optimaler Start ins Leben. Wissenschaftlich erwiesen sind die positiven Effekte auf die Hirnentwicklung, die Immunabwehr und den Schutz vor Darmproblemen besonders bei Frühgeborenen. Für Mütter, die gerade den Schock einer Frühgeburt mit allen ihren Konsequenzen verkraften müssen, ist es zusätzlich belastend, wenn sie keine oder nicht ausreichend eigene Milch für ihre Kinder haben. Hier will die Frauenmilchbank-Initiative (FMBI) Unterstützung bieten.

\section{Bekämpfung der hohen Säuglingssterblichkeit}

Bereits zu Beginn des 20. Jahrhunderts sind die ersten Frauenmilchsammelstellen (FMS) in verschiedenen Städten dokumentiert: 1908/1909 in Wien, 1910 in Boston und im polnischen Lemberg, 1911 in Magdeburg und 1913 in Düsseldorf und New York. In allen Städten war das Ziel durch das Sammeln gespendeter Muttermilch, die damals weltweit sehr hohe Säuglingssterblichkeit zu bekämpfen. 1919 wurde im Krankenhaus Magdeburg-Altstadt die offiziell erste FMS Deutschlands gegründet. Die Kinderärztin Marie-Elise Kayser beschritt dafür neue Wege: Sie warb auch auBerhalb der Klinik und den damals üblichen Wöchnerinnenheimen um Spenderinnen. So konnte sie größere Milchmengen sammeln und die Methode der Pasteurisierung half ihr, eine längere Haltbarkeit zu erzielen. In den 1930er-Jahren wurde das Spenden von Muttermilch zunehmend politisiert und vom NS-Regime instrumentalisiert. Das ging so weit, dass es jüdischen Müttern
1942 explizit verboten wurde, ihre Milch an FMS zu spenden. Nach dem Zweiten Weltkrieg nahmen die FMS in West- und Ostdeutschland ihre Arbeit wieder auf. Doch im Westen Deutschlands machte sich der zunehmende Einfluss der Formula-Nahrung bemerkbar. In den 1970er-Jahren hatten nur einige wenige Kliniken eine Frauenmilchbank für den Eigenbedarf. In der DDR dagegen gab es bis zur Wiedervereinigung bis zu 60 FMS.

\section{Die beste Alternative zu Muttermilch}

Nachdem zahlreiche Studien belegen konnten, dass gerade für extrem unreife Frühgeborene die Ernährung mit Frauenmilch alternativlos ist, empfehlen die Weltgesundheitsorganisation (WHO), der United Nations Children's Fund (UNICEF) und die Europäische Gesellschaft für Pädiatrische Gastroenterologie, Hepatologie und Ernährung (ESPG-HAN) die Ernährung mit Spenderinnenmilch aus Frauenmilchbanken als die beste Alternative, wenn das Baby keine Milch von der eigenen Mutter bekommen kann. So erleben seit den 2010er-Jahren die Frauenmilchbanken in Deutschland eine Renaissance. 2018 wurde die Frauenmilchbank-Initiative (FMBI) in Erfurt als gemeinnütziger Verein gegründet. In der Initiative treffen sich Still- und Laktationsberaterinnen, Neonatolog*innen, Kinderärzt*innen, Pflegepersonal, Hebammen und interessierte Eltern. Sie eint der Wunsch, weitere Frauenmilchbanken (FMB) in Deutschland zu etablieren, da derzeit der Großteil der Perinatalzentren keinen Zugang zu gespendeter Muttermilch hat. So ist ein Hauptziel der FMBI, dass es bis 2023 in jedem Bundesland mindestens eine FMB gibt.

\section{Zahlreiche Projekte}

Neben der Unterstützung beim Aufbau einer FMB, bietet die Initiative auch Informationen in Form von Vorträgen, Seminaren oder Workshops an. Auf Tagungen, Kongressen und Fortbildungen geben die Mitglieder der FMBI ihr Wissen weiter. Viele dieser Vorträge sind auf der Webseite der FMBI abrufbar.
Doch nicht nur der Wissenstransfer ist dem Verein wichtig. Er möchte auch einheitliche Standards etablieren und hat dazu bei der Arbeitsgemeinschaft der Wissenschaftlichen Medizinischen Fachgesellschaften (AWMF) die Erarbeitung einer Leitlinie angeregt.

Seit dem 1. Januar 2021 läuft das vom Innovationsfonds der Bundesregierung geförderte Projekt „Neo-Milk“, an dem die FMBI neben Kliniken, Krankenkassen und wissenschaftlichen Instituten beteiligt ist: Ab dem kommenden Jahr soll deutschlandweit an zwölf Perinalzentren ein Konzept zur Stillförderung umgesetzt und Frauenmilchbanken aufgebaut werden.

\section{Katharina Kerlen-Petri}

KONTAKT

Frauenmilchbank-Initiative e.V. Püttkampsweg 5

22609 Hamburg

Telefon: 04038631459

E-Mail: info@fmbi.de

Internet: www.frauenmilchbank.de

\section{Bibliografie}

Die Hebamme 2021; 34: 73

DOI 10.1055/a-1515-1502

ISSN $0932-8122$

(C) 2021. Thieme. All rights reserved.

Georg Thieme Verlag KG, Rüdigerstraße 14, 70469 Stuttgart, Germany 